\title{
Observer-Based Load Frequency Control for Island Microgrid with Photovoltaic Power
}

\author{
Chaoxu Mu, ${ }^{1}$ Weiqiang Liu, ${ }^{1}$ Wei Xu, ${ }^{2,3}$ and Md. Rabiul Islam ${ }^{4}$ \\ ${ }^{1}$ Tianjin Key Laboratory of Process Measurement and Control, School of Electrical and Information Engineering, Tianjin University, \\ Tianjin 300072, China \\ ${ }^{2}$ State Key Laboratory of Advanced Electromagnetic Engineering and Technology, School of Electrical and Electronic Engineering, \\ Huazhong University of Science and Technology, Wuhan 430074, China \\ ${ }^{3}$ College of Mechanical and Electrical Engineering, Huanggang Normal University, Huanggang 438000, China \\ ${ }^{4}$ Department of Electrical and Electronic Engineering, Rajshahi University of Engineering and Technology, Rajshahi 6204, Bangladesh
}

Correspondence should be addressed to Wei Xu; weixu@hust.edu.cn

Received 28 November 2016; Accepted 5 February 2017; Published 26 April 2017

Academic Editor: Zofia Stasicka

Copyright (c) 2017 Chaoxu Mu et al. This is an open access article distributed under the Creative Commons Attribution License, which permits unrestricted use, distribution, and reproduction in any medium, provided the original work is properly cited.

\begin{abstract}
As renewable energy is widely integrated into the power system, the stochastic and intermittent power generation from renewable energy may cause system frequency deviating from the prescribed level, especially for a microgrid. In this paper, the load frequency control (LFC) of an island microgrid with photovoltaic (PV) power and electric vehicles (EVs) is investigated, where the EVs can be treated as distributed energy storages. Considering the disturbances from load change and PV power, an observer-based integral sliding mode (OISM) controller is designed to regulate the frequency back to the prescribed value, where the neural network observer is used to online estimate the PV power. Simulation studies on a benchmark microgrid system are presented to illustrate the effectiveness of OISM controller, and comparative results also demonstrate that the proposed method has a superior performance for stabilizing the frequency over the PID control.
\end{abstract}

\section{Introduction}

With the technological innovation of modern power systems, renewable energy has been widely incorporated to the power system, such as wind energy and solar energy. Although renewable energy causes less pollution and is energy saving, the large-scale integration of renewable energy would have a significant impact on the power system since renewable energy is not ideal for power generation [1-3].

Load frequency stability is regarded as an indispensable factor when considering the stability of power systems [4]. For a microgrid system, if the imbalance between load consumption and power generation frequently happens, then a load frequency controller is required to have an adequate ability to quickly damp the frequency oscillation $[5,6]$. The traditional load frequency model for the power system is approximated as a linear model near an operation point without the generator dynamics being involved, and the most commonly used frequency control strategy for this kind of linear model is the PID control, as reported in [7]. After the offline tuning of parameters, the PID controller can have a good frequency damping performance within a certain range around the designed operation point [8]. With the nonlinear characteristics of power generation for renewable energy, it may be less effective to the microgrid system, especially when the microgrid system is far away from the set operation point. Besides, several advanced control methods have been used to the LFC problem in the last decade, such as fuzzy logic control [9-11], adaptive control [12, 13], and robust control [14]. Although the design of nonlinear control approaches is relatively complex, the research of advanced nonlinear control approaches on smart grid has been paid great attention since nonlinear control is more close to the characteristics of a power system itself $[3,15-18]$.

In this paper, we investigate the integral sliding mode (ISM) control method for load frequency control of an island 


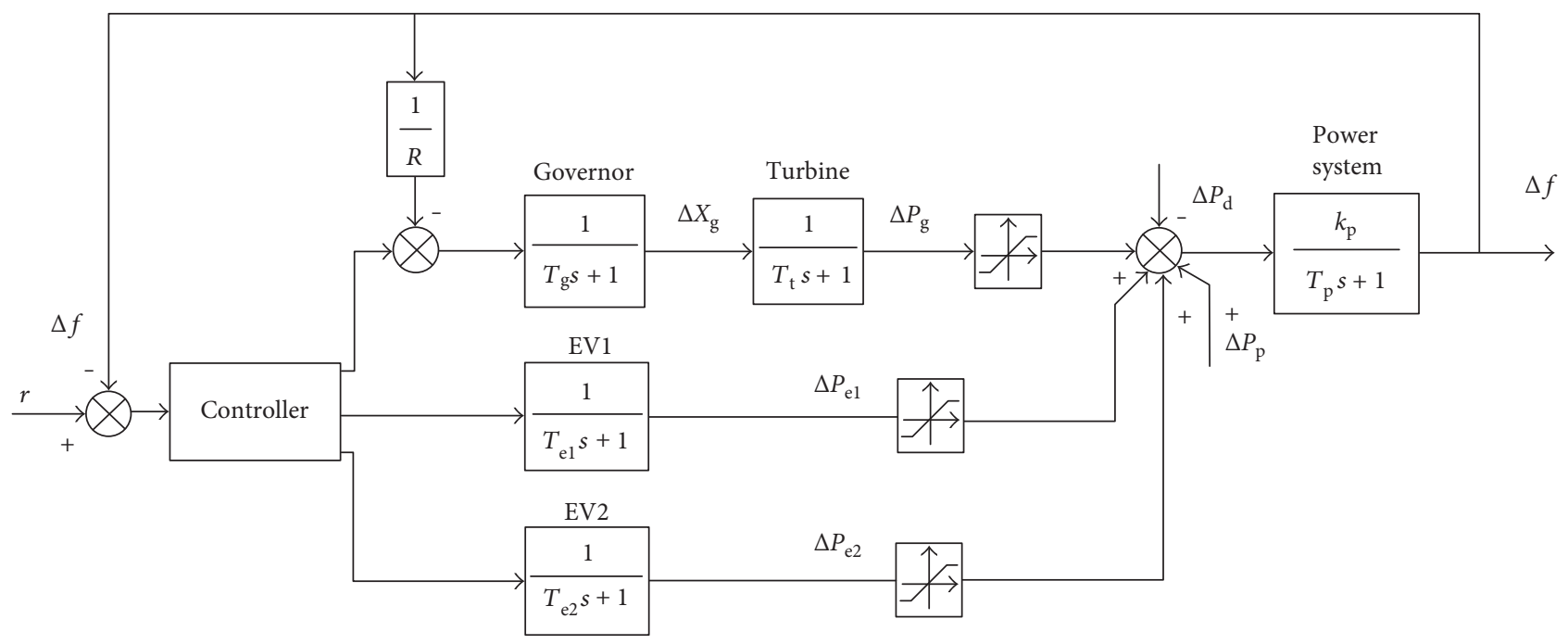

FIgURE 1: The schematic diagram of the benchmark system.

microgrid system with PV power integration, and a neural network observer is designed for an online observation of the PV power generation. Meanwhile, with the improvement of vehicle-to-grid (V2G) technique, EVs are also integrated into the microgrid as distributed storage devices to support the frequency regulation. The major contribution of this paper is as follows. First, an ISM controller for the microgrid with EVs is designed. Concerning the distinguished pattern of PV power generation, an online OISM controller is proposed to regulate the frequency of the microgrid, and the relevant stability proof is rigorously analyzed. Second, the robustness of the proposed controller against system parameter uncertainties are verified, where the control performance of this proposed method is compared with the traditional PID control.

This paper is organized as follows. Section 2 introduces the studied microgrid system and formulates the frequency control problem. Section 3 designs the neural network observer and further presents the OISM controller method. Simulation is carried out on the benchmark microgrid, and all the results are presented and analyzed in Section 4 . Section 5 concludes this paper.

\section{Problem Formulation}

In this paper, the studied benchmark system includes the following general parts: an equivalent microturbine (MT), PV arrays, two equivalent EV models with battery banks, and a demand side such as smart homes and loads. Both island mode and grid-connected mode are the possible operation modes [19]. In this paper, this benchmark power system is considered as a microgrid and operated in an island mode, where the system power flow is balanced by local loads and local power generation. It means that MTs and PV arrays provide active power to balance all local loads, and EV stations can be considered as distributed battery energy storages to compensate the unbalance between power generation and load demand.
The power system is usually considered to be nonlinear and dynamic, but there only exists small load change during its normal operation. As EVs integrated into the benchmark system, the schematic diagram of the benchmark system is presented in Figure 1.

Since the microgrid is in the island operating mode, the LFC capacity is required to be adequate to quickly damp the frequency oscillation. By incorporating the EVs into the microgrid, the system inertia can be increased and the frequency stability can be improved $[20,21]$. To demonstrate the benefit from the technology of V2G, an active power disturbance is added; the frequency dynamics with EVs and without EVs is shown in Figure 2.

The load frequency controller is expected to maintain the command frequency level when the load disturbances appear and renewable energy is incorporated into the gird. In order to formulate the LFC control problem of this microgrid, some mathematical notations are defined as follows: $\Delta f$, $\Delta P_{\mathrm{t}}, \Delta X_{\mathrm{g}}, \Delta P_{\mathrm{e} 1}$, and $\Delta P_{\mathrm{e} 2}$ are the change of the frequency, the turbine power, the governor position valve, the first EV power, and the second $\mathrm{EV}$ power, respectively. $T_{\mathrm{t}}, T_{\mathrm{g}}, T_{\mathrm{el}}$, $T_{\mathrm{e} 2}$, and $T_{\mathrm{p}}$ are the time constants of the turbine, the governor, the first EV, the second EV, and the power system, respectively. $k_{\mathrm{p}}$ and $R$ are the gain of the power system and the speed regulation coefficient, respectively.

Based on Figure 1, the state vector $x(t)$ of the system is defined as

$$
x(t)=\left[\Delta f(t), \Delta P_{\mathrm{t}}(t), \Delta X_{\mathrm{g}}(t), \Delta P_{\mathrm{e} 1}(t), \Delta P_{\mathrm{e} 2}\right]^{T},
$$

and the LFC model is formulated by the following differential equation:

$$
\dot{x}(t)=A x(t)+B u(t)+F\left(\Delta P_{\mathrm{p}}(t)+\Delta P_{\mathrm{d}}(t)\right) .
$$

The system matrix $A$, the control matrix $B$, and the disturbance matrix $F$ can be expressed as 


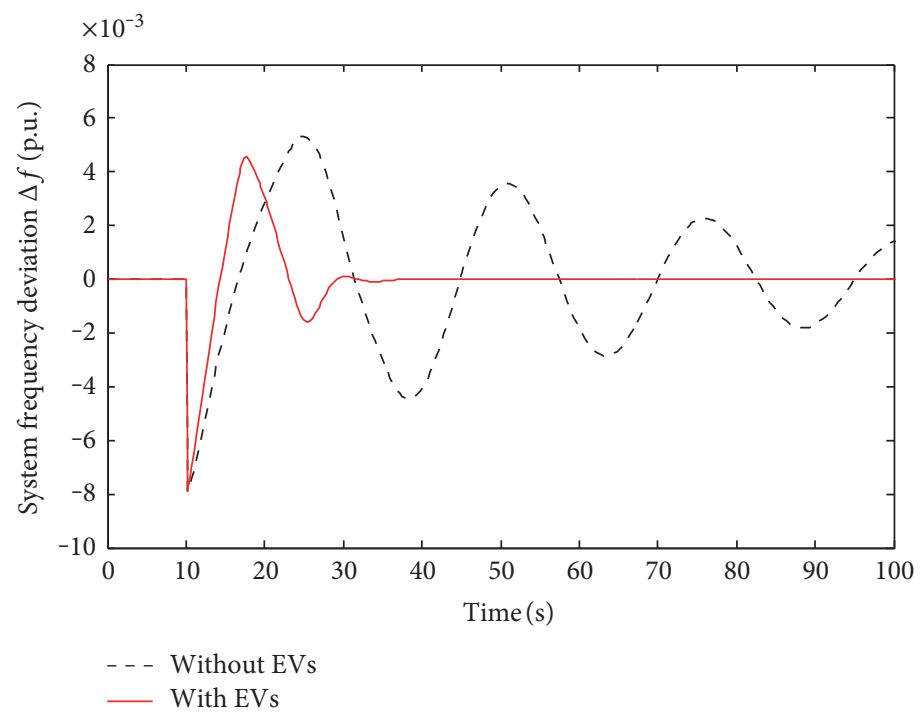

FIgURE 2: The frequency deviation with and without EVs.

$A=\left[\begin{array}{ccccc}-\frac{1}{T_{\mathrm{p}}} & \frac{k_{\mathrm{p}}}{T_{\mathrm{p}}} & 0 & \frac{k_{\mathrm{p}}}{T_{\mathrm{p}}} & \frac{k_{\mathrm{p}}}{T_{\mathrm{p}}} \\ 0 & -\frac{1}{T_{\mathrm{t}}} & \frac{1}{T_{\mathrm{t}}} & 0 & 0 \\ -\frac{1}{R T_{\mathrm{g}}} & 0 & -\frac{1}{T_{\mathrm{g}}} & 0 & 0 \\ 0 & 0 & 0 & -\frac{1}{T_{\mathrm{e} 1}} & 0 \\ 0 & 0 & 0 & 0 & -\frac{1}{T_{\mathrm{e} 2}}\end{array}\right]$,

$B=\left[\begin{array}{ccc}0 & 0 & 0 \\ 0 & 0 & 0 \\ \frac{1}{T_{\mathrm{g}}} & 0 & 0 \\ 0 & \frac{1}{T_{\mathrm{e} 1}} & 0 \\ 0 & 0 & \frac{1}{T_{\mathrm{e} 2}}\end{array}\right]$,

$F=\left[\begin{array}{c}k_{\mathrm{p}} \\ T_{\mathrm{p}} \\ 0 \\ 0 \\ 0 \\ 0\end{array}\right]$

$u(t)$ is the control vector, $u(t)=\left[\Delta u_{1}(t), \Delta u_{2}(t), \Delta u_{3}(t)\right]^{T}$, and $\Delta P_{\mathrm{d}}(t)$ and $\Delta P_{\mathrm{p}}(t)$ are the integrated disturbance from load change and the PV power, respectively. The following assumption is applied on the benchmark system.

Assumption 1. The induced norms of PV power disturbances and load change satisfy $\left\|\Delta P_{\mathrm{p}}(t)\right\| \leq \alpha_{1}$ and $\left\|\Delta P_{\mathrm{d}}(t)\right\| \leq \alpha_{2}$, respectively, where $\alpha_{1} \geq 0$ and $\alpha_{2} \geq 0$ represent upper bounds.

\section{Controller Design}

3.1. Integral Sliding Mode Controller. Compared with a large interconnected system, the microgrid system is more instable and easy to cause serious damage under parameter uncertainties and disturbances. Therefore, a controller with excellent robustness to maintain the microgrid system stable is required.

Sliding mode control is robust and systematic for matched disturbances and parameter variations [22-25]. To keep the frequency in the required level, ISM control is selected as the fundamental control method for the LFC problem of the benchmark system. For the microgrid system presented in (2), an ISM variable is designed as

$$
s(t)=C_{1} x(t)+\int_{0}^{t} C_{2} x(t) d t,
$$

where $C_{1}=\left[c_{1}, c_{2}, c_{3}, c_{4}, c_{5}\right]$ and $C_{2}=\left[c_{6}, c_{7}, c_{8}, c_{9}, c_{10}\right]$ are the coefficient vectors, and $c_{i}$ meets that the two polynomials $c_{5} p^{4}+c_{4} p^{3}+c_{3} p^{2}+c_{2} p+c_{1}$ and $c_{10} p^{4}+c_{9} p^{3}+c_{8} p^{2}+c_{7} p+c_{6}$ are Hurwitz.

The ISM controller is designed by adopting the reaching law $\dot{s}(x)=-\varepsilon s a t(s)$. With the idea of equivalent control, the ISM frequency controller is constituted as

$$
u(t)=-\left(C_{1} B\right)^{-1}\left(C_{1} A x(t)+C_{2} x(t)+\varepsilon s a t(s)\right),
$$

where $\varepsilon$ is the control gain and satisfies $\varepsilon>\left|C_{1} F\left(\alpha_{1}+\alpha_{2}\right)\right|$ and $\operatorname{sat}(s)=s(t) / \rho$ is the saturation function to eliminate the chattering in the control signal by setting a reasonable width $\rho$.

3.2. Neural Network Observer. In this subsection, the data of the PV power is measured by specific sensors and is stored in the control unit. Recently, intelligent algorithms have been developed for obtaining information from data [26-31]. Therefore, these data are available to design an observer to forecast the future PV power. In this paper, a three-layer neural 


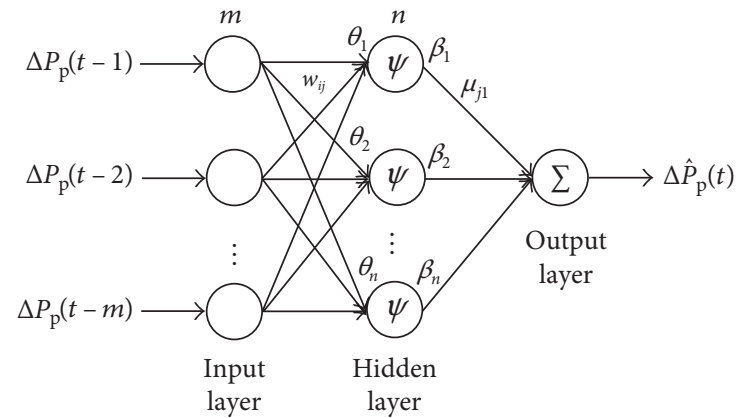

Figure 3: The structure of the neural network observer.

network with a hidden layer is used to realize the online observation of PV power, whose structure is shown in Figure 3.

From Figure 3, we can know that the neural network observer for the PV power is with $m$ input-layer neurons, $n$ hidden-layer neurons, and one output-layer neuron. Therefore, the inputs of the observer are $m$ PV power values before time $t$, recorded as $I_{t} \in R^{1 \times m}$. The output $\Delta P_{\mathrm{p}}(t)$ is the PV power value at time $t$. The rest of the variables in Figure 3 can be defined as

$$
\begin{aligned}
\theta_{j}(t) & =\sum_{i=1}^{m} w_{i j}(t) \Delta P_{\mathrm{p}}(t-i), \\
\beta_{j}(t) & =\psi\left(\theta_{j}(t)\right)=\frac{1-e^{-\theta_{j}(t)}}{1+e^{-\theta_{j}(t)}}, \\
\Delta \widehat{P}_{\mathrm{p}}(t) & =\sum_{j=1}^{n} \mu_{j 1}(t) \beta_{j}(t),
\end{aligned}
$$

where $i$ and $j$ satisfy $i=1, \ldots, m$ and $j=1, \ldots, n$, respectively. $\theta_{j}(t)$ and $\beta_{j}(t)$ are input and output values of the $j$ th hidden neuron, respectively. $w_{i j}(t)$ is the weight from the $i$ th input neuron to the $j$ th hidden neuron, and $\mu_{j 1}(t)$ is the weights from the $j$ th hidden node to the output node. $\psi(z)=\left(1-e^{-z}\right) /\left(1+e^{-z}\right)$ is used as the activation function.

The weight vectors $w_{i j}(t)$ and $\mu_{j 1}(t)$ are randomly initialized in $[-1,1]$. According to the calculation of forward propagation, the neural network outputs $\Delta \widehat{P}_{\mathrm{p}}(t)$ as the estimation value of PV power at time $t$.

The weights of the neural network are updated by the error back-propagation algorithm. The difference between $\Delta \widehat{P}_{\mathrm{p}}(t)$ and the measured real value of $\mathrm{PV}$ power is defined as the network error, which is

$$
e_{\mathrm{p}}(t)=\Delta P_{\mathrm{p}}(t)-\Delta \widehat{P}_{\mathrm{p}}(t)
$$

If $e_{\mathrm{p}}(t)=0$, it represents the estimated PV power value which is completely equal to the real PV power value. In the backpropagation process, the aim is to minimize the objective function $E(t)$ associated with $e_{\mathrm{p}}(t)$; that is,

$$
E(t)=\frac{1}{2} e_{\mathrm{p}}^{2}(t)
$$

TABLE 1: The system parameters of the benchmark system.

\begin{tabular}{lccccccc}
\hline Parameters & $T_{\mathrm{t}}$ & $T_{\mathrm{g}}$ & $T_{\mathrm{e} 1}$ & $T_{\mathrm{e} 2}$ & $T_{\mathrm{p}}$ & $k_{\mathrm{p}}$ & $R$ \\
\hline Values & 10 & 0.1 & 1 & 1 & 10 & 1 & 0.5 \\
\hline
\end{tabular}

TABLE 2: The disturbance from load change.

\begin{tabular}{lcccc}
\hline Time (s) & 15 & 60 & 100 & 150 \\
\hline Disturbance (p.u.) & +0.2 & -0.2 & -0.4 & +0.4 \\
\hline
\end{tabular}

The gradient descent method is used to update the weights of the neural network observer in the backpropagation [26-28, 32]. Define the input-to-hidden weight vector $w_{1}(t)$ and the hidden-to-output weight vector $w_{2}(t)$ as

$$
\begin{aligned}
& w_{1}(t)=\left\{w_{i j}(t) \mid i=1,2, \ldots, m, j=1,2, \ldots, n\right\}, \\
& w_{2}(t)=\left\{\mu_{j 1}(t) \mid j=1,2, \ldots, n\right\},
\end{aligned}
$$

where $w_{1}(t) \in R^{m \times n}$ and $w_{2}(t) \in R^{n \times 1}$, respectively. Then (8) can be further simplified as

$$
\Delta \widehat{P}_{\mathrm{p}}(t)=\sum_{j=1}^{n} \mu_{j 1}(t) \psi\left(\sum_{i=1}^{n} w_{i j}(t) \Delta P_{\mathrm{p}}(t-i)\right)=w_{2}(t) \psi\left(w_{1}\right) .
$$

According to the chain derivation rule, the weights of the neural network observer are updated by

$$
\dot{w}_{2}(t)=-\tau \frac{\partial E(t)}{\partial \Delta \widehat{P}_{\mathrm{p}}(t)} \frac{\partial \Delta \widehat{P}_{\mathrm{p}}(t)}{\partial w_{2}(t)}=\tau e_{\mathrm{p}}(t) \psi\left(w_{1}\right) .
$$

Specifically, the weights $w_{i j}(t)$ and $\mu_{j 1}(t)$ are regulated by

$$
\begin{aligned}
& \dot{w}_{i j}(t)=-\tau \frac{\partial E(t)}{\partial \Delta \widehat{P}_{\mathrm{p}}(t)} \frac{\partial \Delta \widehat{P}_{\mathrm{p}}(t)}{\partial \alpha_{j}(t)} \frac{\partial \alpha_{j}(t)}{\partial \theta_{j}(t)} \frac{\partial \theta_{j}(t)}{\partial w_{i j}(t)}, \\
& \dot{\mu}_{j 1}(t)=-\tau \frac{\partial E(t)}{\partial \Delta \widehat{P}_{\mathrm{p}}(t)} \frac{\partial \Delta \widehat{P}_{\mathrm{p}}(t)}{\partial \mu_{j 1}(t)}
\end{aligned}
$$

where $\tau>0$ is the learning rate. The estimated PV power can be used as an input signal into the ISM controller to eliminate the influence of integrated PV power.

3.3. Observer-Based Integral Sliding Mode Controller. According to the universal approximation property of neural networks $[32,33]$, the output of the observer can approximate the real PV power with an allowable error. In other words, there exists a weight vector $w$ to make the error $e_{\mathrm{p}}(t)$ reach the minimum, which is

$$
\left|e_{\mathrm{p}}(t)\right| \leq \xi
$$

where $\xi$ is an arbitrary enough small positive constant. It represents the maximal absolute difference between the 


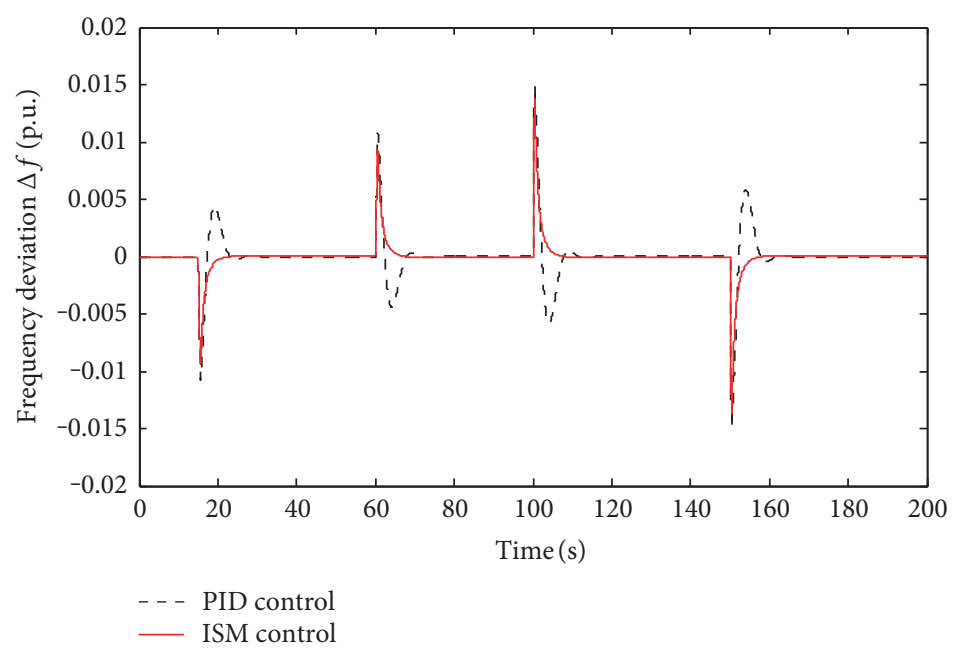

FIgURE 4: The frequency deviation without power output constraints.
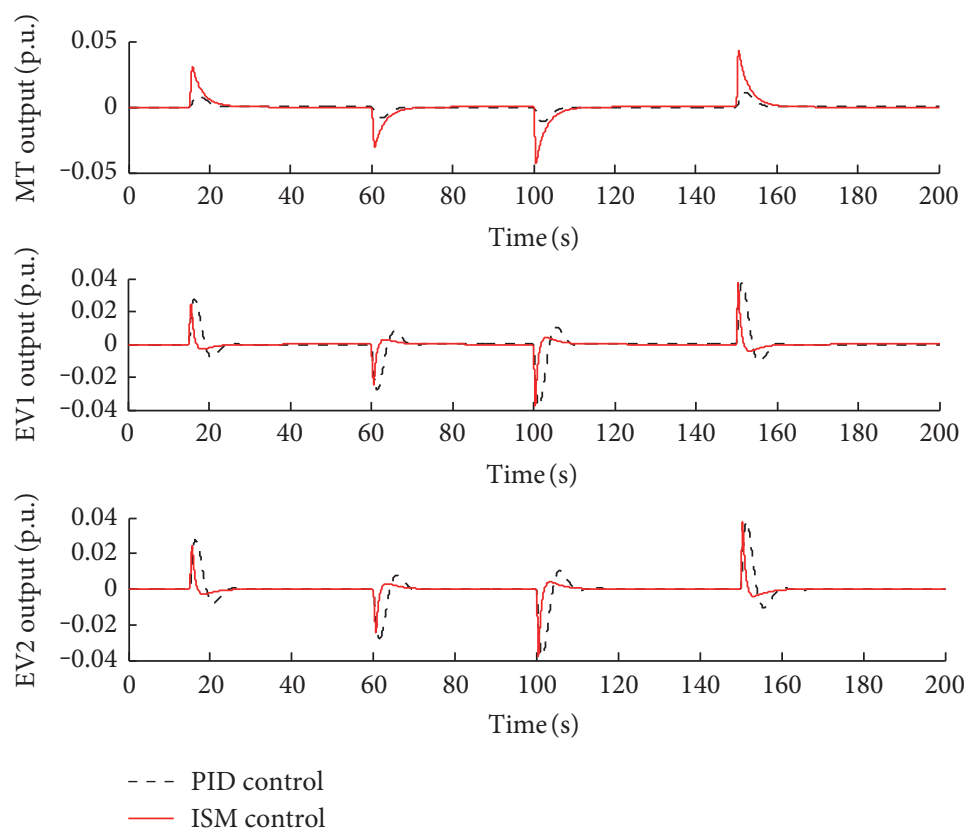

Figure 5: The output power of MT and EVs without power output constraints.

estimated value and the real PV power. By introducing the neural network observer, the OISM controller is constructed for the microgrid system with PV power integration. Thus, we have the following theorem.

Theorem 1. For system (1), if the weights of the neural network observer are updated by (13), and the OISM controller is designed as

$\mu(t)=-\left(C_{1} B\right)^{-1}\left(C_{1} A x(t)+C_{2} x(t)+\varepsilon s a t(s)+C_{1} F \Delta \widehat{P}_{\mathrm{p}}(t)\right)$,

then the microgrid system is asymptotically stable.
Proof. We define the Lyapunov function for system (1) with control law (17) as

$$
V(t)=\frac{1}{2} s^{2}(t)+\frac{1}{2 \tau} \tilde{w}_{2}^{2}(t) .
$$

It is obvious that $V(t) \geq 0$. Set the weight error vector as

$$
\tilde{w}_{2}(t)=w_{2}^{*}(t)-w_{2}(t)
$$

where $w_{2}^{*}(t)$ is the ideal weight vector and $w_{2}(t)$ is actually weight vector in the observer. By differentiating $\tilde{w}_{2}(t)$ with respect to time $t$, we could obtain

$$
\dot{\tilde{w}}_{2}(t)=-\dot{w}_{2}(t) .
$$




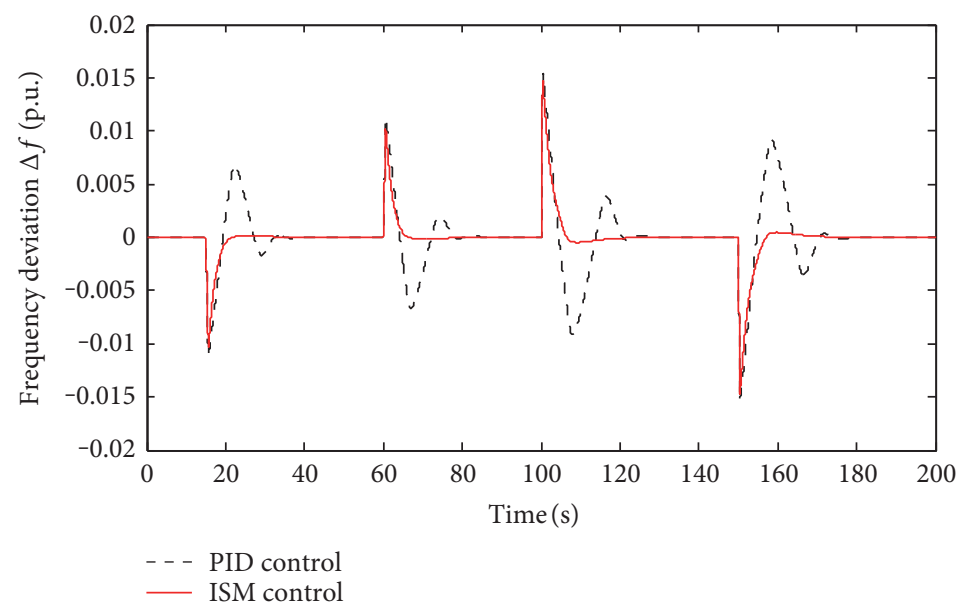

FIGURE 6: The frequency deviation with power output constraints.
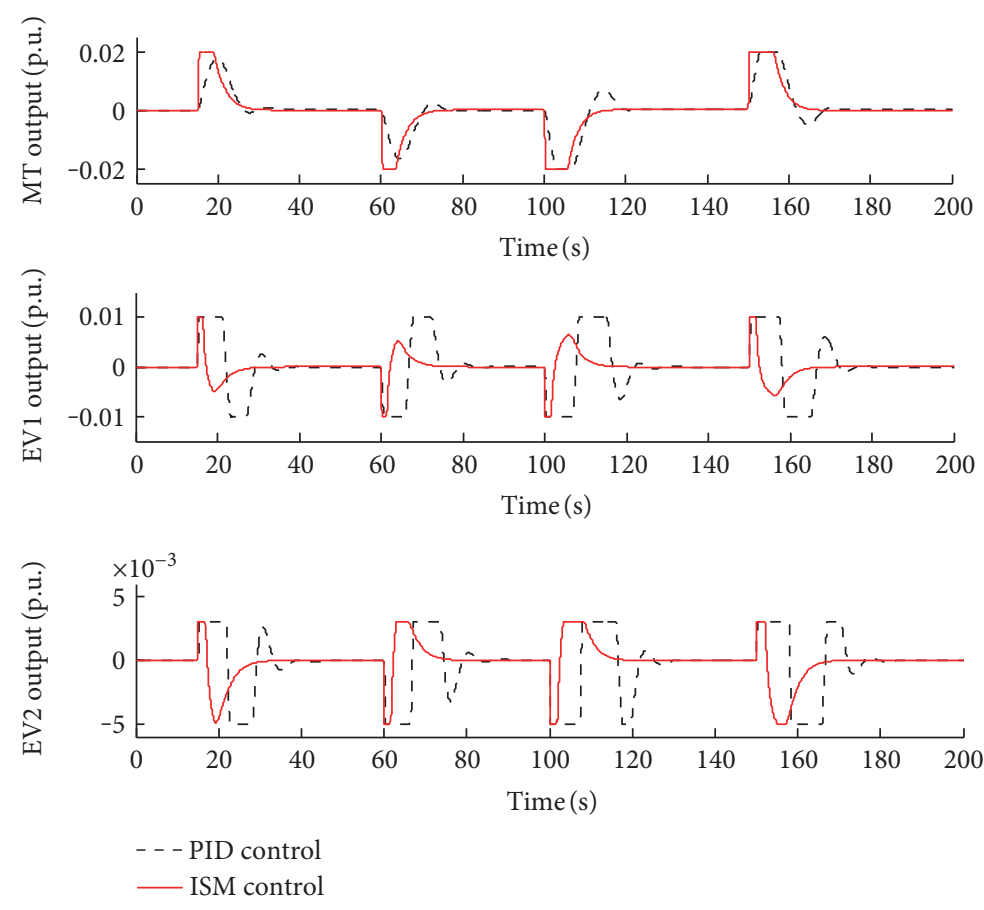

FIgURE 7: The output power of MT and EVs with power output constraints.

TABLE 3: The sum of frequency deviation.

\begin{tabular}{lcccc}
\hline Condition & PID & ISM & PID + constraints & ISM + constraints \\
\hline$E_{\text {iae }}$ & 0.1309 & 0.066 & 0.3251 & 0.1212 \\
\hline
\end{tabular}

By combining with (20), the derivative of $V(t)$ can be obtained as

$$
\begin{aligned}
\dot{V}(t) & =s(t) \dot{s}(t)+\tau^{-1} \tilde{w}_{2}(t) \tilde{w}_{2}(t) \\
& =s(t) \dot{s}(t)-\tau^{-1} \tilde{w}_{2}(t) \dot{w}_{2}(t)
\end{aligned}
$$

According to (2) and (4), then $\dot{V}(t)$ can be deduced as

$$
\dot{V}(t)=s(t)\left(C_{1} \dot{x}(t)+C_{2} x(t)\right)-\tau^{-1} \tilde{w}_{2}(t) \dot{w}_{2}(t) .
$$

The weights of the neural network observer are updated according to (13); then $\dot{V}(t)$ can be derived as

$$
\begin{aligned}
\dot{V}(t)=s(t)\left(C_{1} A x(t)\right. & +\left(-C_{1} A x(t)-C_{2} x(t)-\varepsilon s a t(s)-C_{1} F \Delta \widehat{P}_{s}(t)\right) \\
& \left.+C_{1} F\left(\Delta P_{s}(t)+\Delta P_{\mathrm{d}}(t)\right)+C_{2} x(t)\right) \\
& +\tau^{-1} \tilde{w}_{2}(t) \dot{w}_{2}(t) .
\end{aligned}
$$

We simplify (23) and substitute $\tilde{w}_{2}(t)=w_{2}^{*}(t)-w_{2}(t)$; then $\dot{V}(t)$ becomes 


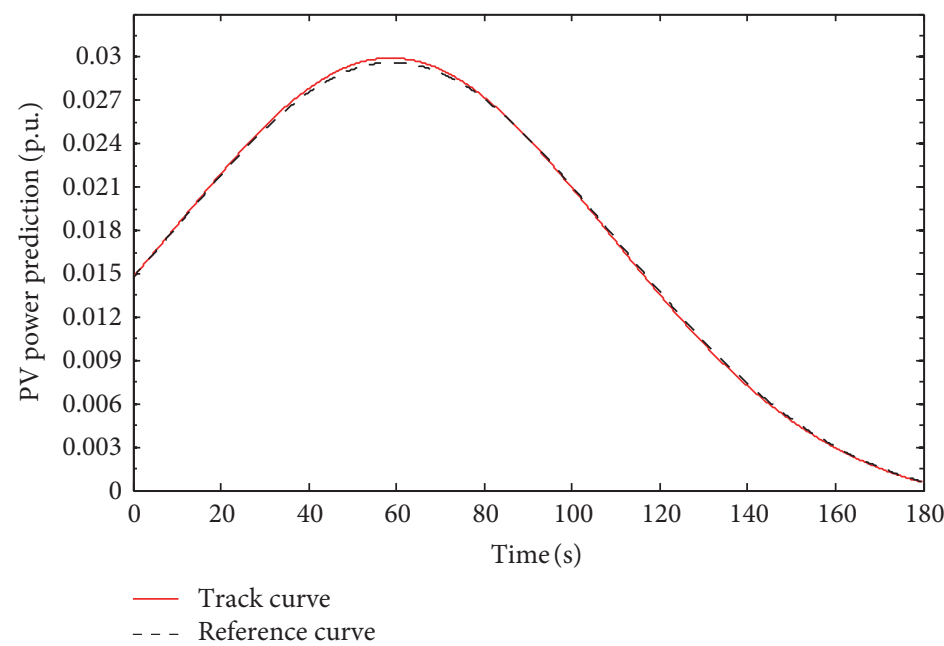

FIGURE 8: The predictive PV power by the neural network observer.

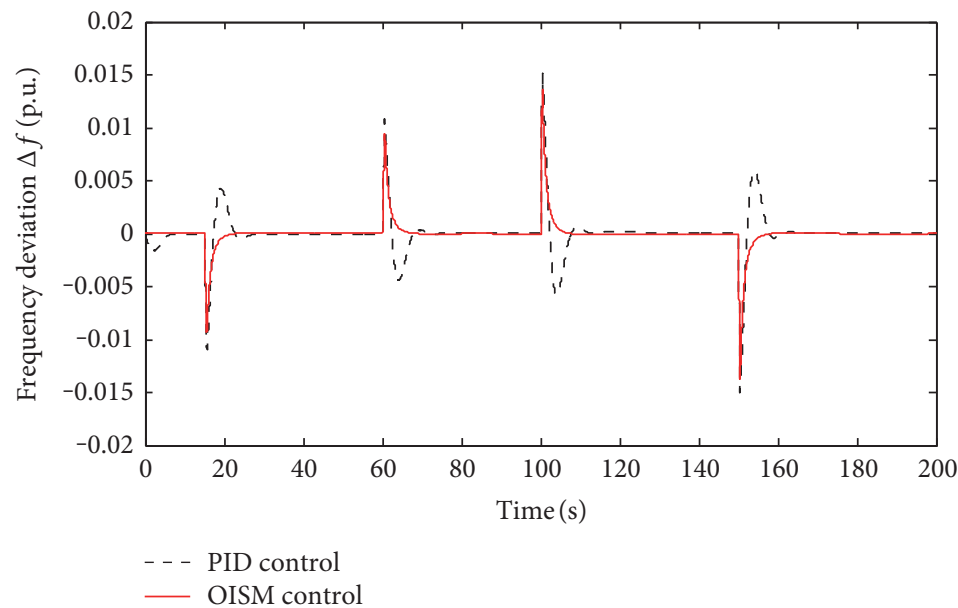

FIGURE 9: The frequency performance without power output constraints.

$$
\begin{aligned}
\dot{V}(t) & =s(t)\left(-\varepsilon s a t(s)+C_{1} F\left(e_{\mathrm{p}}(t)+\Delta P_{\mathrm{d}}(t)\right)\right) \\
& -\left(w_{2}^{*}-w_{2}\right)(t) \psi\left(w_{1}(t) I_{t}\right) e_{\mathrm{p}}(t)
\end{aligned}
$$

According to the universal approximation property of neural network and Assumption 1, that is, $\left|e_{\mathrm{p}}(t)\right|$ $<\xi,\left|\Delta P_{\mathrm{p}}(t)\right| \leq \alpha_{1}$, and $\left|\Delta P_{\mathrm{d}}(t)\right| \leq \alpha_{2}$, then we can obtain that the sliding mode gain $\varepsilon$ is required to satisfy

$$
\varepsilon \geq\left|C_{1} F\left(\alpha_{1}+\alpha_{2}\right)\right| \text {. }
$$

After a sufficient learning, the error of the neural network observer is reasonable to be small enough, which means $\xi \leq \alpha_{1}$. Therefore, it is easy to deduce

$$
-\varepsilon \leq C_{1} F\left(e_{\mathrm{p}}(t)+\Delta P_{\mathrm{d}}(t)\right) \leq \varepsilon .
$$

With $w_{2}^{*} \psi\left(w_{1}\right)=\Delta P_{p}(t)$ and $w_{2} \psi\left(w_{1}\right)=\Delta \widehat{P}_{p}(t)$, therefore we have the following:

$$
\dot{V}(t)=s(t)\left(-\varepsilon s a t(s)+C_{1} F\left(e_{\mathrm{p}}(t)+\Delta P_{\mathrm{d}}(t)\right)\right)-e_{\mathrm{p}}^{2}(t) .
$$

It means

$$
\dot{V}(t) \leq-e_{\mathrm{p}}^{2}(t)
$$

for both $s(t)>0$ and $s(t)<0$.

Observing inequalities (18) and (28), controller (17) can ensure $V(t) \geq 0$ and $\dot{V}(t) \leq 0$ for system (1). According to the Lyapunov stability theorem, system (1) is asymptotically stable. It means the frequency derivation of system (1) is regulated to zero under the designed OISM controller.

\section{Simulation and Analysis}

In this section, we apply the proposed frequency control method to the studied benchmark microgrid, which is a typical benchmark system. The parameters of a benchmark 

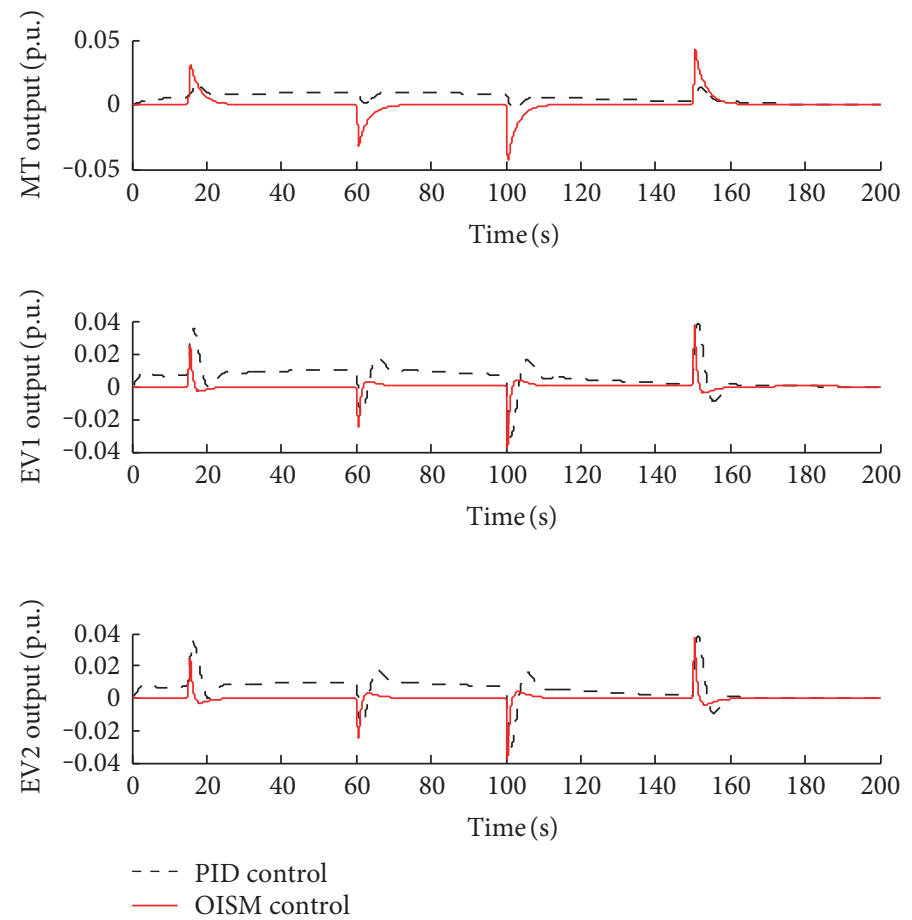

FIgUre 10: The output power of MT and EVs without power output constraints.

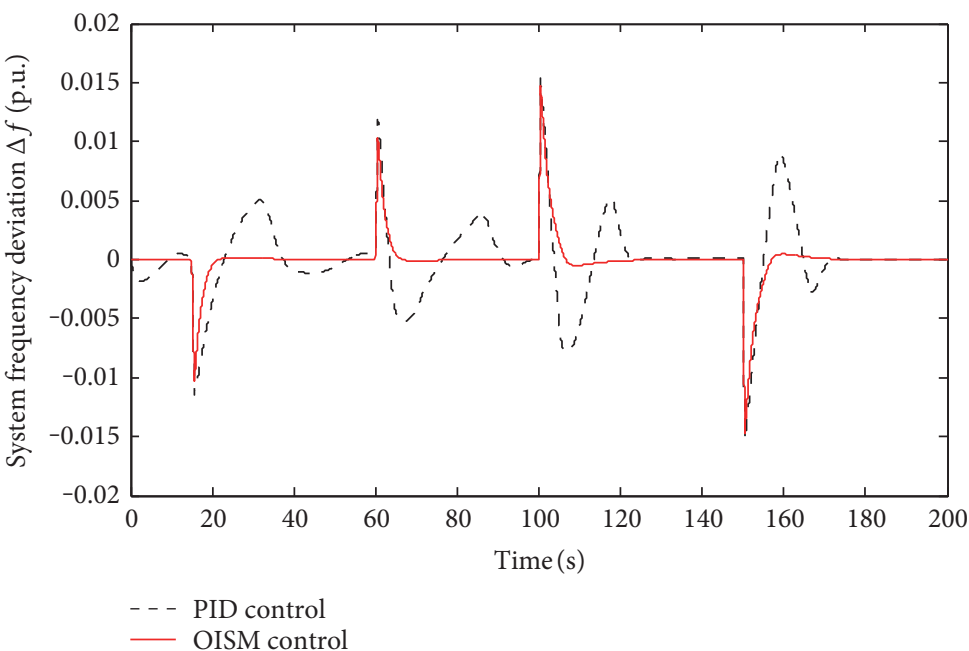

FIGURE 11: The frequency performance with power output constraints.

microgrid system are given in Table 1 by referring to $[19,34,35]$. The PID controller is also used to damp the frequency deviation of the benchmark system as a competitive method.

In the PID controller, it takes the frequency deviation $\Delta f(t)$ as the input, and the parameters of proportional, integral, and derivative gains are set as $K_{\mathrm{p}}=4, K_{\mathrm{i}}=1$, and $K_{\mathrm{d}}=0.6$, respectively. For the proposed ISM control method, the control gain is $\varepsilon=10$, and the coefficient vectors $C_{1}$ and $C_{2}$ are defined as $C_{1}=[0.65,0.05,0.05,0.05,0.05]$ and $C_{2}=[0.15,0.01,0.01,0.01,0.01]$, respectively. In order to validate the performance of designed controllers, we have the following cases.

4.1. Dynamic Response for Load Change. In this case, the frequency of the microgrid system is initially stable; four sequential active power disturbances caused by load change are applied to the microgrid system, which is shown in Table 2. Without integrating the PV power, the ISM controller in (5) is used to suppress the frequency oscillation.

When these sequential disturbances are added on the system, the performance of the integral sliding mode controller 


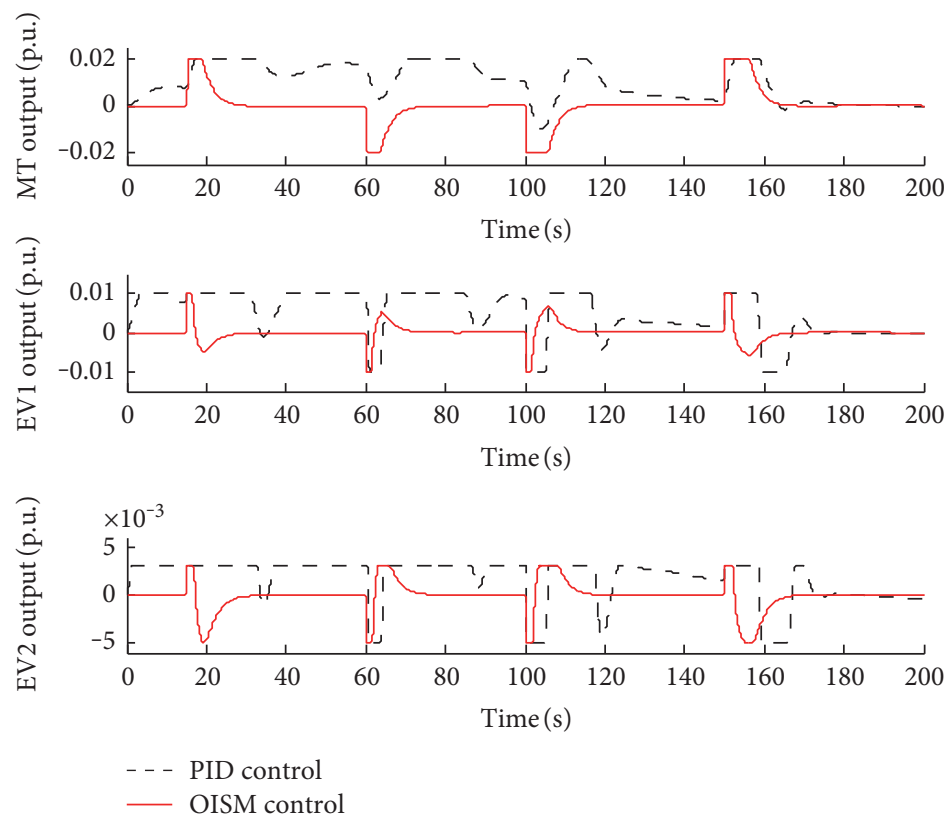

FIGURE 12: The frequency performance with power output constraints.

TABLE 4: The sum of frequency deviation.

\begin{tabular}{lcccc}
\hline & PID & OISM & PID + constraints & OISM + constraints \\
\hline$E_{\text {iae }}$ & 0.1459 & 0.0679 & 0.4102 & 0.1230 \\
\hline
\end{tabular}

is presented in Figure 4. As a competitive result, the regulation result of PID controller is also presented in Figure 4. The power outputs of MT and EVs are shown in Figure 5.

\subsection{Dynamic Response to Load Disturbances and Power} Output Constraints. Consider the output power limits of MT and two EVs as [36]. The output power values of MT and two EVs have output limits which are $[-0.02,+0.02]$, $[-0.01,+0.01]$, and $[-0.005,+0.003]$ for the output power ranges of MT, EV1, and EV2, respectively. The same disturbances given in Table 2 are added on this benchmark system. In this case, the performance of system frequency deviation is shown in Figure 6, including the PID control and the ISM control. The outputs of MT and EVs are shown in Figure 7.

From Figures 4 and 6, we can observe that every disturbance generates an obvious frequency deviation from the prescribed value, and the deviation depends on the amplitude of disturbance. Compared with the PID controller, the ISM controller has a faster response ability as well as smaller transient amplitudes. Moreover, without the power output constraints, the regulation of both PID and ISM controller has better performance than the two controllers under the condition of power output constraints. Figures 5 and 7 show the power outputs of MT and EVs without and with constraints.

The performance index based on the integral value of total frequency deviation in the whole regulation process is defined as

$$
E_{\text {iae }}=\int_{0}^{t}|\Delta f| d t
$$

For the above two cases, the performance indexes of two controllers are presented in Table 3. Comparatively speaking, the ISM controller has a better regulation ability than the PID control.

4.3. Dynamic Response to Both Load Disturbances and PV Power. In this case, the PV power is integrated into the microgrid system based on case 1 . The PV power and four sequential power disturbances are all applied to the microgrid. Specifically, four sequential power disturbances from load changes are given in Table 2.

The parameters of PID controller and ISM controller are the same as the values in case 1 . In order to eliminate the impact of PV power, we change the ISM controller to the OISM controller, which is designed in (17). The parameters of the neural network observer are set as follows: input layer nodes $m=35$, hidden layer nodes $n=6$, and the learning rate $\tau=0.01$. It means that the neural network learns from 35 previous values before time $t$ and then estimates the PV power at time $t$. The output of the neural network observer is shown in Figure 8, compared with the real PV power. From Figure 8, we can know that the neural network observer is able to predict the PV power well by learning the previous $\mathrm{PV}$ power data. 
After integrating the PV power into the system and applying these sequential disturbances, the system frequency deviation curves with PID controller and OISM controller are presented in Figure 9, and the output power of MT and EVs are shown in Figure 10.

4.4. Dynamic Response to Load Disturbances, PV Power, and Power Output Constraints. Considering the output power limits of MT and two EVs, the output constraints of MT and EVs use the same settings as case 2. The applied disturbances are also shown in Table 2. By using the OISM controller, the system frequency deviation is shown in Figure 11, and the outputs of MT and EVs are shown in Figure 12. The performance of PID controller has been provided as the comparison.

From Figures 9 and 11, the frequency deviates from the command value under the disturbances from PV power and load change. However, the OISM controller is more effective than the PID controller, which can provide the control performance with faster regulation and smaller oscillation in the whole regulation process. From Figures 10 and 12 , we can know that the PID controller hardly adjusts the deviation of the output power to zero; it means that the frequency is difficult to be regulated to the specified value. Comparing the control performance with the PID controller, the OISM controller still keeps fast regulation and strong robustness with an observed PV power. Similar to case 2, the performance of the PID controller becomes worse when the output power is constrained. From Figures 11 and 12, we can observe that it is hard for the PID controller to regulate the frequency to the required value under disturbances. On the contrary, the OISM controller still has a superior damping performance.

The performance index $E_{\text {iae }}$ is also used to evaluate the control performance. The index values of the controllers are presented in Table 4. It can be seen that the performance of the OISM controller is obviously better than that of the PID controller for the whole regulation process.

\section{Conclusions}

This paper has addressed the LFC problem for the island microgrid with EVs and PV power integration. The OISM control strategy is adopted to regulate the frequency derivation, where the neural network observer is designed to predict the PV power disturbance. The theoretical analysis is presented for the OISM system in the sense of Lyapunov stability. Simulations have been executed on a benchmark system with different conditions, including load change, PV disturbances, and power output limits. Comparative studies with the PID controller are provided to demonstrate the superior performance of the OISM controller. In the future work, there are several significant topics that need to be intensively addressed. For example, integrating the diversified sources of energy into the island microgrid will be considered. Also, we will consider the coordination control of diversified energies and the frequency stability of interconnected microgrids.

\section{Conflicts of Interest}

The authors declare that they have no conflicts of interest.

\section{Acknowledgments}

This work was supported in part by the National Natural Science Foundation under Grants 51377065, 61304018, 61301035, and 6141130160, in part by Hubei Province Science and Technology Supporting Program under Grant 2014BAA035, and in part by Tianjin Natural Science Foundation under Grant 14JCQNJC05400.

\section{References}

[1] J. A. P. Lopes, F. J. Soares, and P. M. R. Almeida, "Integration of electric vehicles in the electric power system," Proceedings of the IEEE, vol. 99, no. 1, pp. 168-183, 2011.

[2] Y. Lei, W. Xu, C. Mu, Z. Zhao, H. Li, and Z. Li, "New hybrid damping strategy for grid-connected photovoltaic inverter with LCL filter," IEEE Transactions on Applied Superconductivity, vol. 24, no. 5, pp. 1-8, 2014.

[3] C. Mu, Y. Tang, and H. He, "Observer-based sliding mode frequency control for micro-grid with photovoltaic energy integration," 2016 IEEE Power and Energy Society General Meeting (PESGM), pp. 1-5, 2016.

[4] C. T. Pan and C. M. Liaw, "An adaptive controller for power system load-frequency control," IEEE Transactions on Power Systems, vol. 4, no. 1, pp. 122-128, 1989.

[5] X. Li, Y.-J. Song, and S.-B. Han, "Frequency control in microgrid power system combined with electrolyzer system and fuzzy PI controller," Journal of Power Sources, vol. 180, no. 1, pp. $468-475,2008$.

[6] I. Kamwa, R. Grondin, and Y. Hebert, "Wide-area measurement based stabilizing control of large power systems-a decentralized/hierarchical approach," IEEE Transactions on Power Systems, vol. 16, no. 1, pp. 136-153, 2001.

[7] P. Kundur, Power System Stability and Control, McGraw-Hill Education, New York, 1st edition, 1994.

[8] Y. Tang, J. Yang, J. Yan, and H. He, "Intelligent load frequency controller using GrADP for island smart grid with electric vehicles and renewable resources," Neurocomputing, vol. 170, pp. 406-416, 2015.

[9] M. H. Ali, T. Murata, and J. Tamura, "Transient stability enhancement by fuzzy logic-controlled SMES considering coordination with optimal reclosing of circuit breakers," IEEE Transactions on Power Systems, vol. 23, no. 2, pp. 631-640, 2008.

[10] E. Cam and I. Kocaarslan, "Load frequency control in two area power systems using fuzzy logic controller," Energy Conversion and Management, vol. 46, no. 2, pp. 233-243, 2005.

[11] I. Kocaarslan and E. Cam, "Fuzzy logic controller in interconnected electrical power systems for load-frequency control," International Journal of Electrical Power \& Energy Systems, vol. 27, no. 8, pp. 542-549, 2005.

[12] M. Zribi, M. Al-Rashed, and M. Alrifai, "Adaptive decentralized load frequency control of multi-area power systems," International Journal of Electrical Power \& Energy Systems, vol. 27, no. 8, pp. 575-583, 2005.

[13] A. Rubaai and V. Udo, "An adaptive control scheme for load-frequency control of multiarea power systems part I. 
Identification and functional design," Electric Power Systems Research, vol. 24, no. 3, pp. 183-188, 1992.

[14] S. Vachirasricirikul and I. Ngamroo, "Robust LFC in a smart grid with wind power penetration by coordinated V2G control and frequency controller," IEEE Transactions on Smart Grid, vol. 5, no. 1, pp. 371-380, 2014.

[15] S. Saxena and Y. V. Hote, "Load frequency control in power systems via internal model control scheme and model-order reduction," IEEE Transactions on Power Systems, vol. 28, no. 3, pp. 2749-2757, 2013.

[16] X. Liu, X. Kong, and K. Y. Lee, "Distributed model predictive control for load frequency control with dynamic fuzzy valve position modelling for hydro-thermal power system," IET Control Theory and Applications, vol. 10, no. 14, pp. 1653-1664, 2016.

[17] K. Vrdoljak, N. Peric, and I. Petrovic, "Sliding mode based load-frequency control in power systems," Electric Power Systems Research, vol. 80, no. 5, pp. 514-527, 2010.

[18] Y. Tang, C. Mu, and H. He, "SMES-based damping controller design using fuzzy-GrHDP considering transmission delay," IEEE Transactions on Applied Superconductivity, vol. 26, no. 7, pp. 1-6, 2016.

[19] D. Qian, S. Tong, H. Liu, and X. Liu, "Load frequency control by neural-network-based integral sliding mode for nonlinear power systems with wind turbines," Neurocomputing, vol. 173, Part 3, pp. 875-885, 2016.

[20] S. D. G. Jayasinghe, D. M. Vilathgamuwa, and U. K. Madawala, "Direct integration of battery energy storage systems in distributed power generation," IEEE Transactions on Energy Conversion, vol. 26, no. 2, pp. 677-685, 2011.

[21] L. A. de Souza Ribeiro, O. R. Saavedra, S. L. de Lima, and J. G. de Matos, "Isolated microgrids with renewable hybrid generation: the case of Lencois Island," IEEE Transactions on Sustainable Energy, vol. 2, no. 1, pp. 1-11, 2011.

[22] B. Bandyopadhyay, F. Deepak, and K.-S. Kim, Sliding Mode Control Using Novel Sliding Surfaces, Springer-Verlag, Berlin Heidelberg, 2009.

[23] V. I. Utkin, Sliding Modes in Control and Optimization, Springer-Verlag, Berlin Heidelberg, 1992.

[24] C. Mu, W. Xu, and C. Sun, "On switching manifold design for terminal sliding mode control," Journal of the Franklin Institute, vol. 353, no. 7, pp. 1553-1572, 2016.

[25] C. Mu, Z. Ni, C. Sun, and H. He, "Air-breathing hypersonic vehicle tracking control based on adaptive dynamic programming," IEEE Transactions on Neural Networks and Learning Systems, vol. 28, no. 3, pp. 584-598, 2017.

[26] L. Cheng, Z.-G. Hou, and M. Tan, “Adaptive neural network tracking control for manipulators with uncertain kinematics, dynamics and actuator model," Automatica, vol. 45, no. 10, pp. 2312-2318, 2009.

[27] D. Wang, D. Liu, C. Mu, and H. Ma, "Decentralized guaranteed cost control of interconnected systems with uncertainties: a learning-based optimal control strategy," Neurocomputing, vol. 214, pp. 297-306, 2016.

[28] D. Wang, C. Li, D. Liu, and C. Mu, "Data-based robust optimal control of continuous-time affine nonlinear systems with matched uncertainties," Information Sciences, vol. 366, pp. 121-133, 2016.
[29] B. Gu, V. S. Sheng, K. Y. Tay, W. Romano, and S. Li, "Incremental support vector learning for ordinal regression," IEEE Transactions on Neural Networks and Learning Systems, vol. 26, no. 7, pp. 1403-1416, 2015.

[30] B. Gu, V. S. Sheng, Z. Wang, D. Ho, S. Osman, and S. Li, "Incremental learning for v-support vector regression," Neural Networks, vol. 67, pp. 140-150, 2015.

[31] B. Gu, X. Sun, and V. S. Sheng, "Structural minimax probability machine," IEEE Transactions on Neural Networks and Learning Systems, 2016, in press.

[32] D. Wang, C. Mu, and D. Liu, "Data-driven nonlinear near-optimal regulation based on iterative neural dynamic programming," Acta Automatica Sinica, vol. 43, no. 3, pp. $366-375,2017$.

[33] K. Hornik, "Approximation capabilities of multilayer feedforward networks," Neural Networks, vol. 4, no. 2, pp. 251-257, 1991.

[34] Y. Mi, Y. Fu, C. Wang, and P. Wang, "Decentralized sliding mode load frequency control for multi-area power systems," IEEE Transactions on Power Systems, vol. 28, no. 4, pp. 4301-4309, 2013.

[35] C. Wang, Y. Mi, Y. Fu, and P. Wang, "Frequency control of an isolated micro-grid using double sliding mode controllers and disturbance observer," IEEE Transactions on Smart Grid, 2016, in press.

[36] J. Yang, Z. Zeng, Y. Tang, J. Yan, H. He, and Y. Wu, "Load frequency control in isolated micro-grids with electrical vehicles based on multivariable generalized predictive theory," Energies, vol. 8, no. 3, pp. 2145-2164, 2015. 

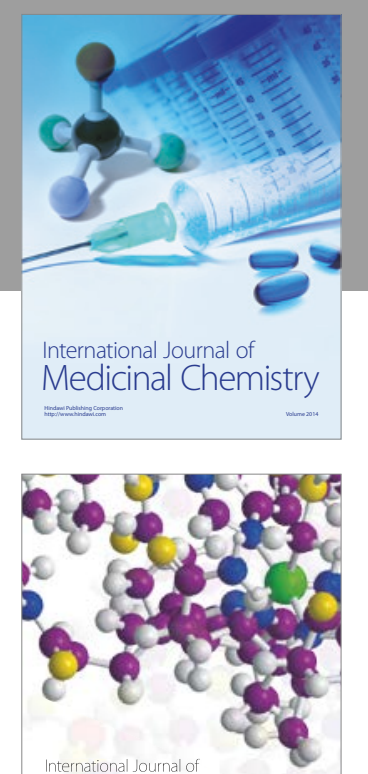

Carbohydrate Chemistry

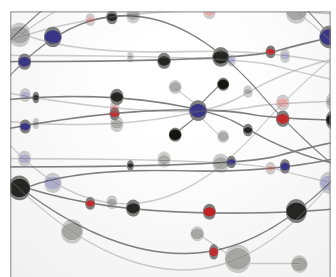

The Scientific World Journal
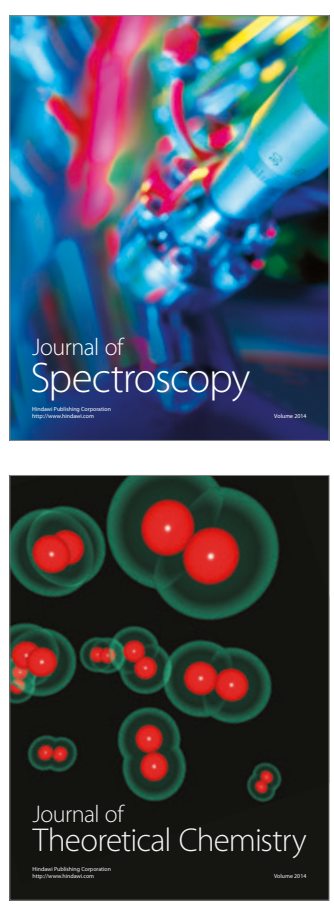
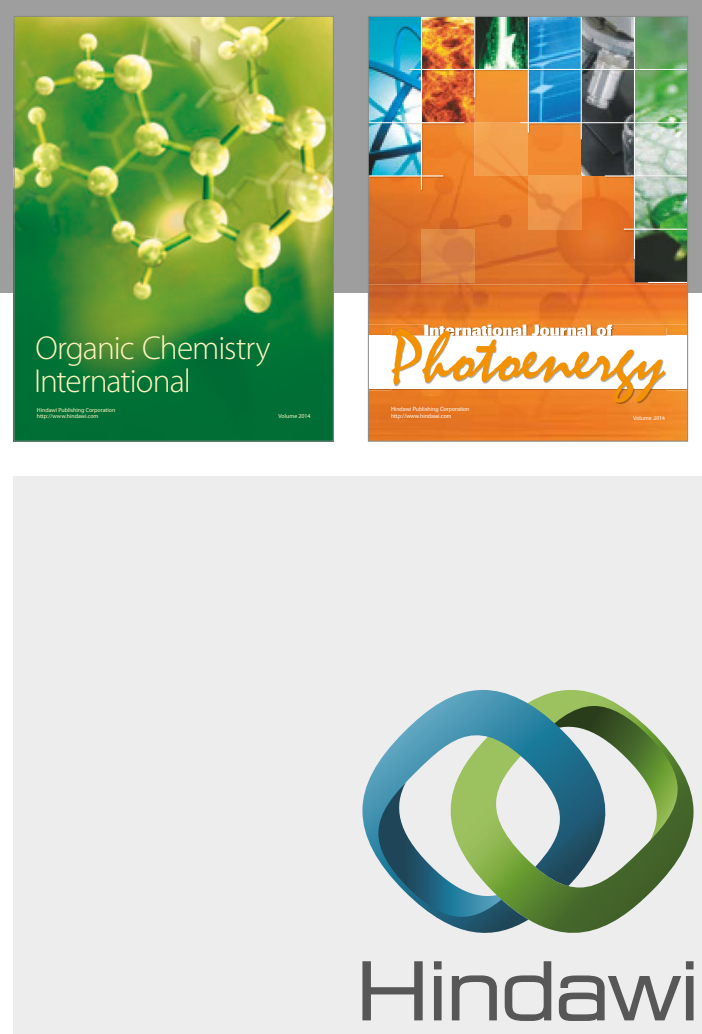

Submit your manuscripts at

https://www.hindawi.com

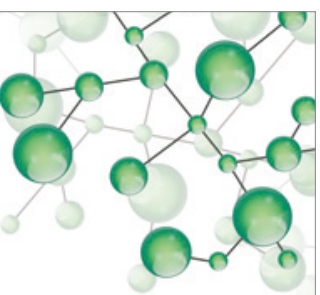

International Journal of

Inorganic Chemistry

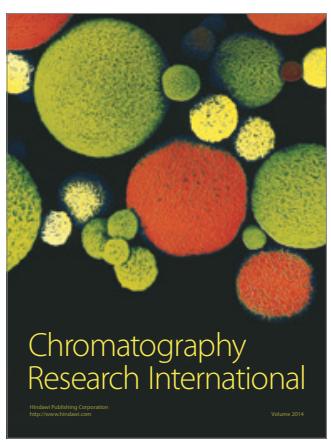

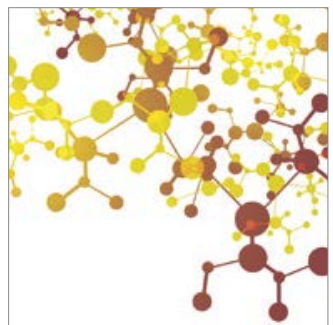

Applied Chemistry
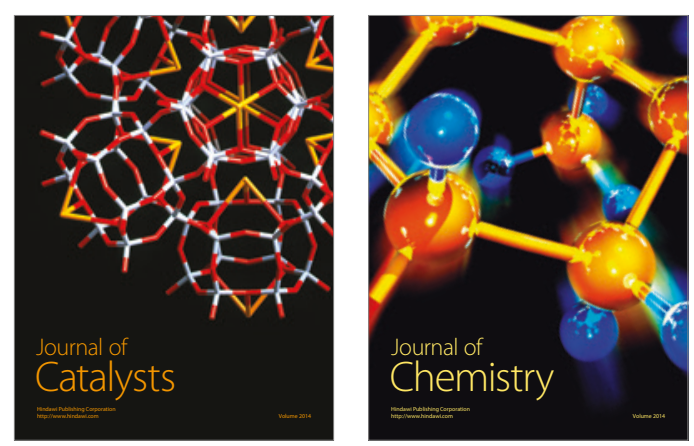
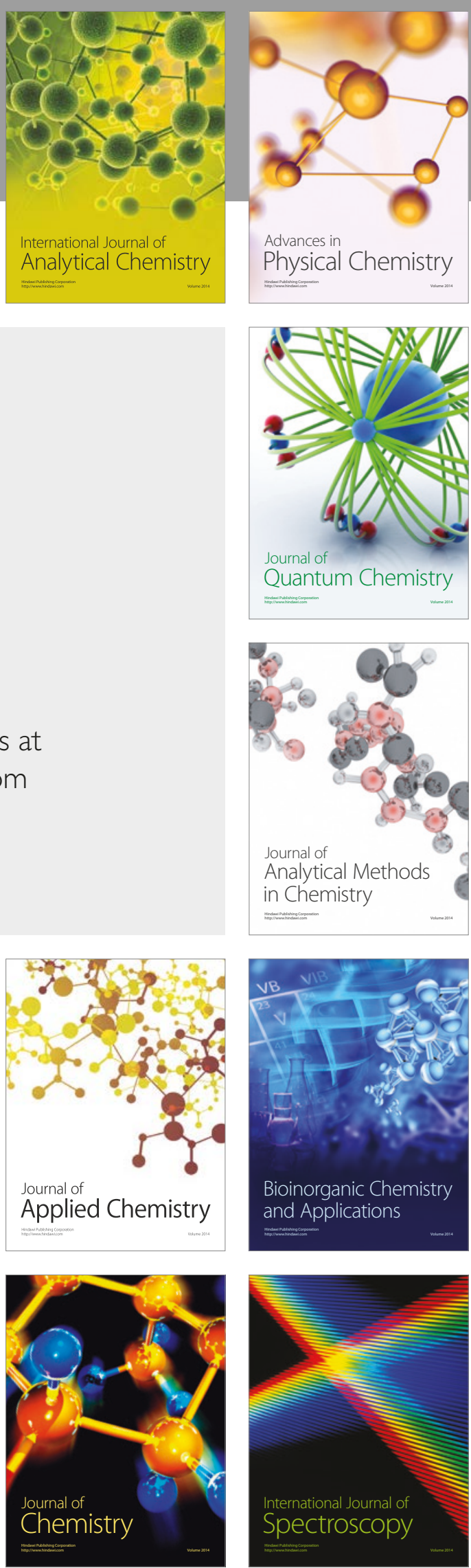